\title{
Multistate Outcome and Persistent Benefit Analysis in Bipolar Mood Disorders
}

JM Azorin Marseille ${ }^{1}$, Charles L Bowden ${ }^{2 *}$, G Perugi $^{3}$, E Vieta $^{4}$ and AH Young ${ }^{5}$

${ }^{1}$ France Hospital de Sainte-Marguerite, Psychiatric University Hospital, Switzerland

${ }^{2}$ University of Texas Health Science Center, San Antonio, USA

${ }^{3}$ University of Pisa, Italy

${ }^{4}$ Hospital Clinic at the University of Barcelona, Spain

${ }^{5}$ Institute of Mental Health, University of British Columbia, Canada

Submission: July 12, 2018; Published: July 23, 2018

*Corresponding author: Charles L Bowden, Chief Scientific Officer, Biomedical Development Corporation, San Antonio, Texas, USA,

Email: bowden4307@gmail.com

Subjects per Country in the N=5635 BRIDGE Study

To Identify Symptoms, Family History and Illness Course Differentiating BD and MDD (Figure 1).

HCL-32 item Frequencies across cultures (BRIDGE) (Figure 2).

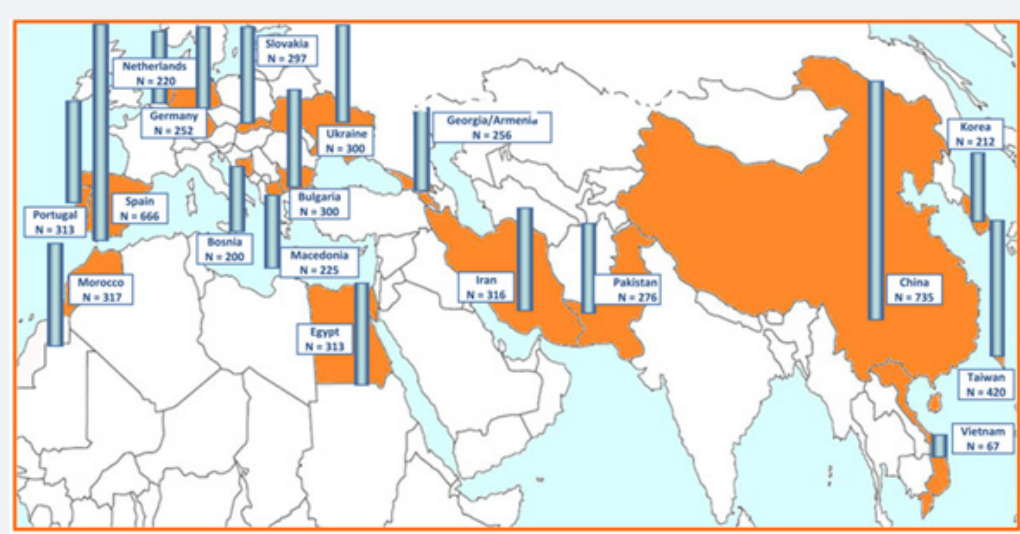

Figure 1: Subjects per Country in the N=5635 BRIDGE Study.

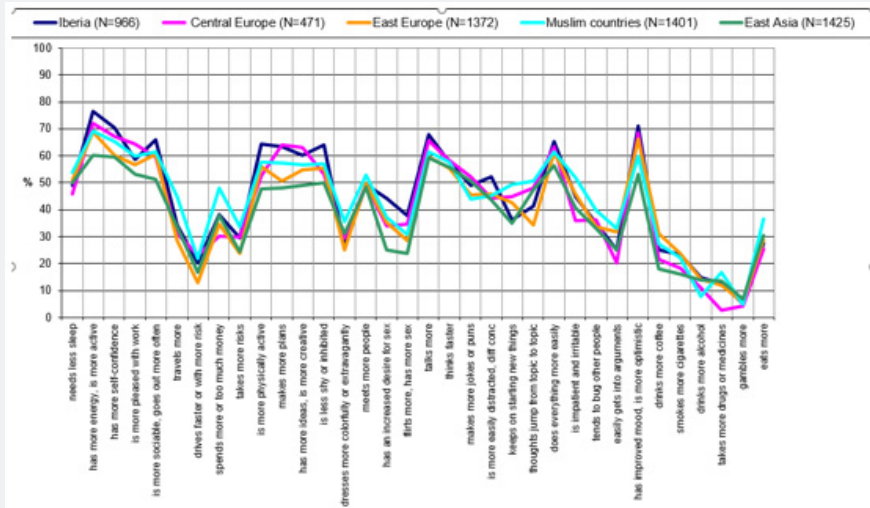

Figure 2: HCL-32 item frequencies across cultures (BRIDGE). 


\section{Global Journal of Intellectual \& Developmental Disabilities}

\section{Data Collected in Bridge}

Patient with major depressive episode meeting DSMIV criteria. Patient age first psychiatric symptoms, age first depression, postpartum depression, suicidal attempts, seasonality of mood episodes, in the past, response to antidepressants,15 a priori predictor variables. Current episode: duration, presence of current depressive symptoms and other psychiatric symptoms. Presence of current comorbid psychiatric conditions according to DSM IV. Global assessment of functioning scale. Hypomania self-rating scale (HCL-32 R2) [1].

\section{BISS in Bipolar}

BISS covers the full spectrum of symptoms seen in this complex condition, including some symptoms that ICD 10 and DSM 5 do not recognize: anxiety, bipolar sleep characteristics. If something is missing from this site that could serve your clinical or research interests, let us know. BISS is a family of scales. Though the main goal is better assessing outcomes with treatments during studies, BISS serves other purposes, such as a quick, self-assessment (KIOS BD). We, and other users, can benefit from learning how a pt. is doing with BISS. We put highlights of this information on the web page. Charles $\mathrm{L}$. Bowden, M.D.

\section{Problems Posed by DSM5 and ICD10 for Biological Research}

Lack primary/secondary symptom distinction. Episode lengths often shorter than DSM requires Hierarchical principle is flawed (elation, grandiosity). Syndromal type weakly predicts duration, course. Structuring clinical trials around DSM diagnoses impedes analyses of neurobiological systems with specific behavioral effects, which could elucidate mechanisms that underlie the efficacy of these agents [2] (Table 1).
Table 1: Thresholds for syndromal or subsyndromal severity.

\begin{tabular}{|c|c|c|}
\hline \multicolumn{3}{|c|}{ Severity Threshold } \\
\hline BISS-15 Scale & Syndromal & Subsyndromal \\
\hline Depression & $\geq 6$ & $\geq 4$ \\
\hline Mania & $\geq 7$ & $\geq 4$ \\
\hline Anxiety domain & $\geq 1.5$ & \\
\hline Irritability domain & $\geq 1.5$ & \\
\hline Psychosis domain & $\geq 1.0$ & \\
\hline
\end{tabular}

\section{Methodologies to Improve Treatment Studies}

Scales covering spectrum of bipolar symptoms, yielding domain scores to profile behavioral targets of drugs. Collaborative studies, compared with single site studies, improve ecological validity, sample size \& control confounds. Improved signal detection by employing innovative statistical methods, e.g., mixed effect, repeat measures > last observations carried forward [3].

\section{Negative Results with SSRIs in Bipolar Depression}

Paroxetine less effective than quetiapine, more associated with switching. Adjunctive paroxetine or imipramine no better than Li alone. Meta-analysis of all types of antidepressant studies showed moderate reduced risk of recurrent depression (0.73) but increased risk of switch to manic episode (1.72) [4].

\section{STEP-BD and Antidepressant Use}

Antidepressants did not adjunctively benefit mood stabilizers for depression. Adding antidepressants increased manic symptoms 3 months later. Even in pts responding initially to antidepressants added to MS, 1-3-year depressive or manic outcomes were not better with continuation of the AD [5-7].

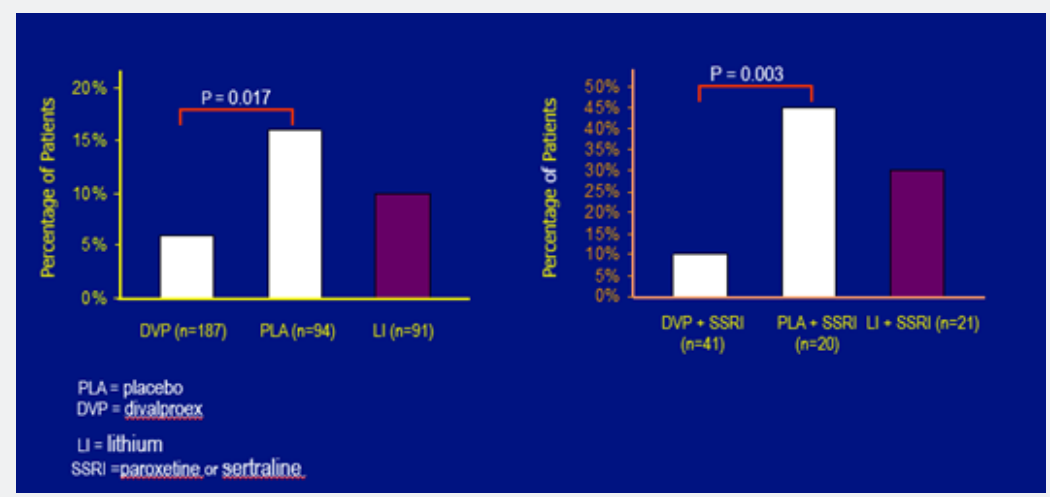

Figure 3: Divalproex + SSRI superior to SSRI in BP I pts who developed depression in 1 yr. study [8].

Divalproex + SSRI superior to SSRI in BP I pts who developed depression in 1 yr. study

(Figure 3).

\section{BISS Affective Lability}

Reported and observed spontaneous sharp shifts of affective or mood states lasting from minutes to hours.
Lability may or may not be influenced by circumstances [8]. Often described metaphorically: "I am on a roller coaster, I am like a yo yo." Affects can be sad, giddy, angry, or over confident. Do not rate on basis of what affective states are expressed. Rate on proportion of time with lability, degree of inability to control affective shifts. How steady has your mood been? Do your emotions shift fairly suddenly at times? Mild; e.g., frequent mild 
mood instability or lability which family, close associates see as dysfunctional [9].
Other Variables that were different with Bipolarity vs Unipolar Depressive Episode

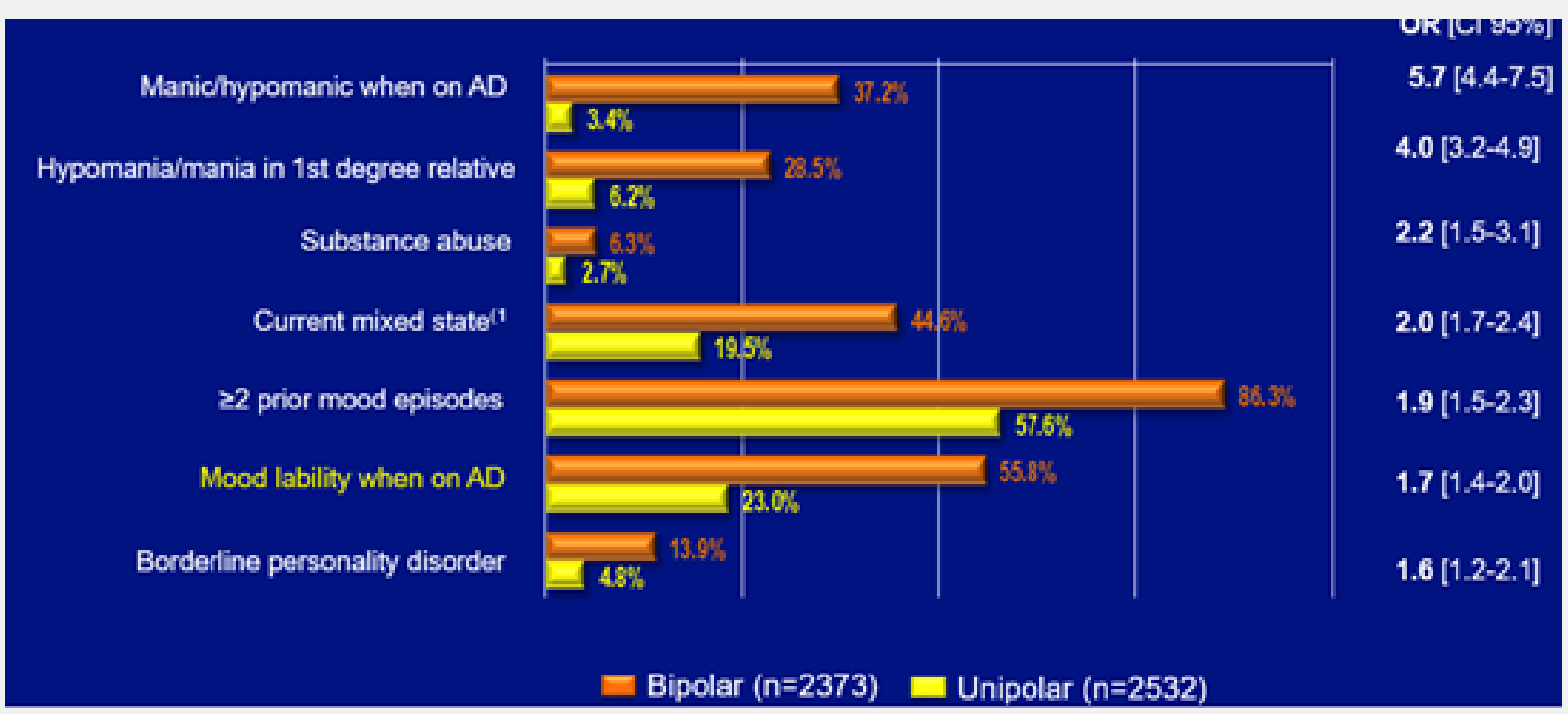

Figure 4: Other Variables that were different with Bipolarity vs Unipolar Depressive Episode [1].

(Figure 4).

\section{Valproate-Lamotrigine Bipolar Depression Study} Conclusions

Mood instability is a core component of bipolar depression, not only manic states, and is not effectively treated by lamotrigine. Valproate plus lamotrigine reduced depressive worsening in treatment of bipolar depression. Study designs that retain high proportions of subjects allow insights into illness course and drug effects that are lost in survival analyses and LOCF imputation $[10,11]$.

Factor Analysis of Lamotrigine Impact in 3 Registration Bipolar Depression Trials

i. Lamotrigine more effective than placebo for depressive cognitions and psychomotor slowing.

ii. Depressive cognitions: mood, guilt, suicidal thoughts, helplessness, hopelessness, worthlessness (HAMD).

iii. Psychomotor slowing : psychomotor retardation, retardation-psychic, retardation-motoric items.

iv. Lam did not benefit insomnia, somatic complaints, anxiety, insomnia, irritability [12].

Bipolar Disorder is Dimensional and Shifting in Severity Over Time

i. Depressive symptoms (32\%) more frequent than manic/hypomanic symptoms (9\%) or mixed (6\%).

ii. Subsyndromal more frequent than syndromal symptoms (30\% vs $11 \%$ ). iii. Bipolar-I patients changed symptom status an average of 6 times per year.

iv. Bipolar disorder is dimensional both in severity and symptom mix [13].

\section{BISS Impulsivity Item}

\section{Impulsive}

Makes decisions without considering consequences. May be associated with intrusiveness, spending, criticism of others, sexual behavior. Have you been patient or impatient when you have had to stand in line, say at a check-out counter? Have you bought things that you did not need? Have you talked when you should have kept quiet? Have you been able to control your responses when placed in a situation.

Design contributors to low generalizability of maintenance studies

i. Monotherapy regimens are generally less effective than combinations (Geddes 2010).

ii. Enriched adjunctive regimens which report low completion rates.

iii. Kaplan Meier Survival analysis, which reports only time to event (the when) such as drop out but does not provide data on whether the event occurred $[14,15]$.

Relationship between onset of anxiety disorders and hypomania

Figure 5. 


\section{Global Journal of Intellectual \& Developmental Disabilities}

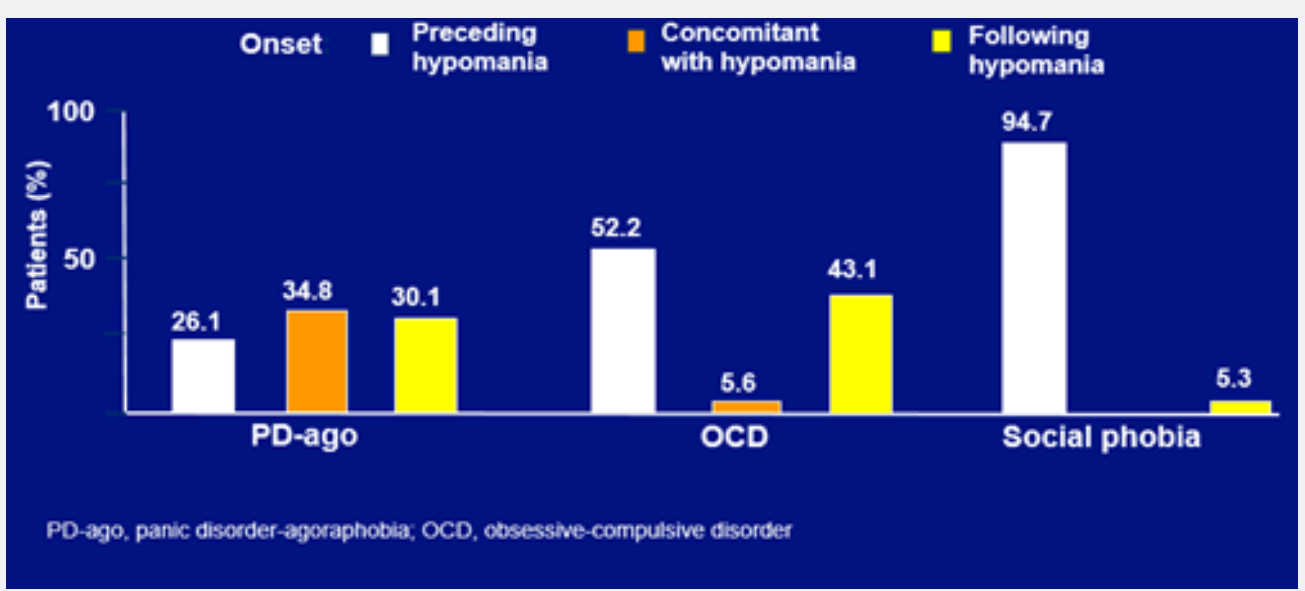

Figure 5: Relationship between onset of anxiety disorders and hypomania [16].

\section{And Yet}

None of the most frequently used scales for mania has an item for anxiety. DSM-V does not include anxiety (or reduced or increased sexual drive) as criteria for bipolar syndromes. The most frequently used depression scale for bipolar disorder has one anxiety item, \& no reduced social or sexual drive item [1618].

\section{BISS Items Loading on Anxiety Factor (Table 2)}

Table 2: BISS Items Loading on Anxiety Factor [17].

\begin{tabular}{|c|c|}
\hline Anxiety observed & .70 \\
\hline Anxiety reported & .59 \\
\hline Fearfulness & .52 \\
\hline Worry & .36 \\
\hline
\end{tabular}

Behavioral Domains from the BISS (Table 3)

Table 3: Behavioral Domains from the BISS [17].

\begin{tabular}{|c|c|}
\hline & Percent of variance accounted for \\
\hline Depression & $15.40 \%$ \\
\hline Mania & $14.00 \%$ \\
\hline Anxiety & $12.10 \%$ \\
\hline
\end{tabular}

\begin{tabular}{|c|c|}
\hline Irritability & $11.80 \%$ \\
\hline Psychosis & $6.00 \%$ \\
\hline$\sum$ variance & $59.30 \%$ \\
\hline
\end{tabular}

Factor analysis, $\mathrm{N}=246,28 \%$ mixed, $27 \%$ depressed, $17 \%$ manic, $7 \%$ subsyndromal, $21 \%$ recovered.

\section{Usefulness of Scales to Clinicians}

Remind us of domains to cover beyond DSM. Allow us to use responses to aid in differential diagnosis within and across disorders. Aid in formulating questions about critical symptom areas. Affective lability. Reduced sex drive.

\section{STEP-BD and Antidepressant Use}

Antidepressants did not adjunctively benefit mood stabilizers (MS) for depression. Adding antidepressants increased manic symptoms 3 months later. Even in pts responding initially to antidepressants added to MS, 1-3-year depressive or manic outcomes were not better with continuation of the AD [5-7].

Effect Sizes by MADRS/BISS-D or MRS/BISS-M Change in Bipolar I and II Patients after $\geq 4$ wks. of Treatment: Influence of Baseline Severity

Figure 6.
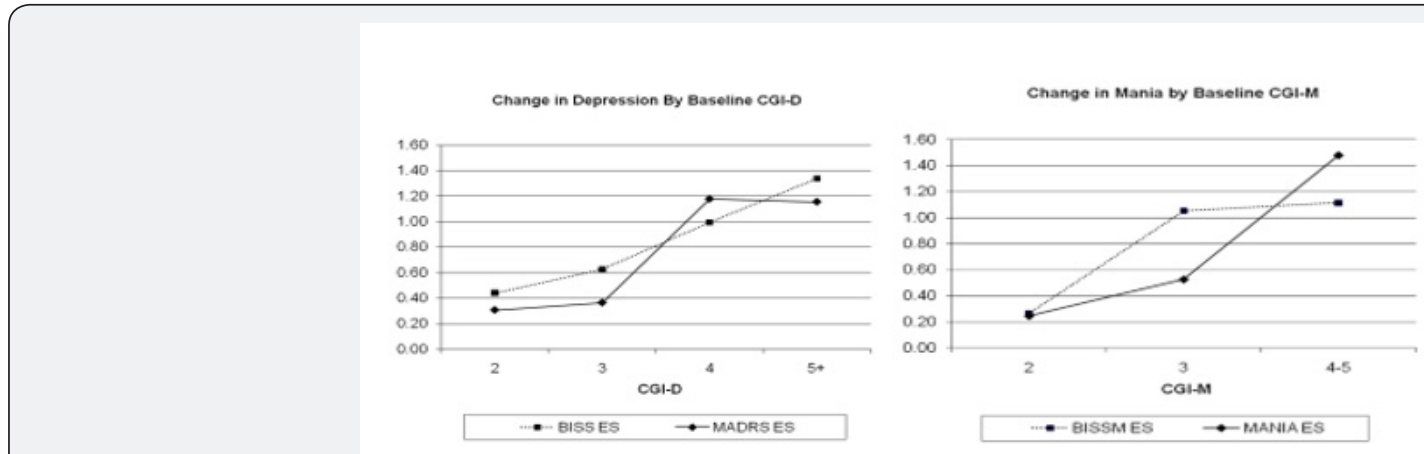

Figure 6: Effect Sizes by MADRS/BISS-D or MRS/BISS-M Change in Bipolar I and II Patients after $\geq 4$ wks. of Treatment: Influence of Baseline Severity [18] 


\section{Global Journal of Intellectual \& Developmental Disabilities}

Multistate Outcome Analysis of Treatment in Bipolar Disorder (MOAT-BD)

Total time spent on drug is partitioned into distinct mood states. Yields remitted, syndromal and subsyndromal manic, depressed mixed states. Estimates total duration in days spent in each mood state by medication group. Provides quantity and quality of time [19].

\section{MOAT Estimates of Mean State Durations}

(Figures 7 \& 8)

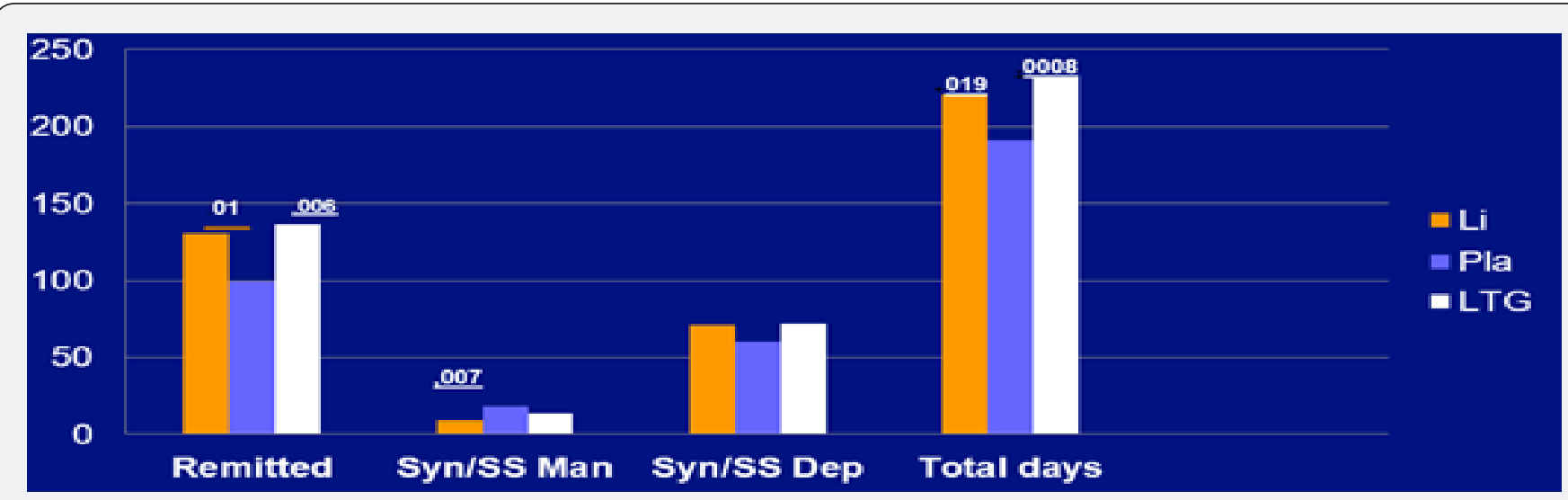

Figure 7: Combined Analysis of LTG, Li and Pla in Manic and Depressed Patients Sample: 578 [19].

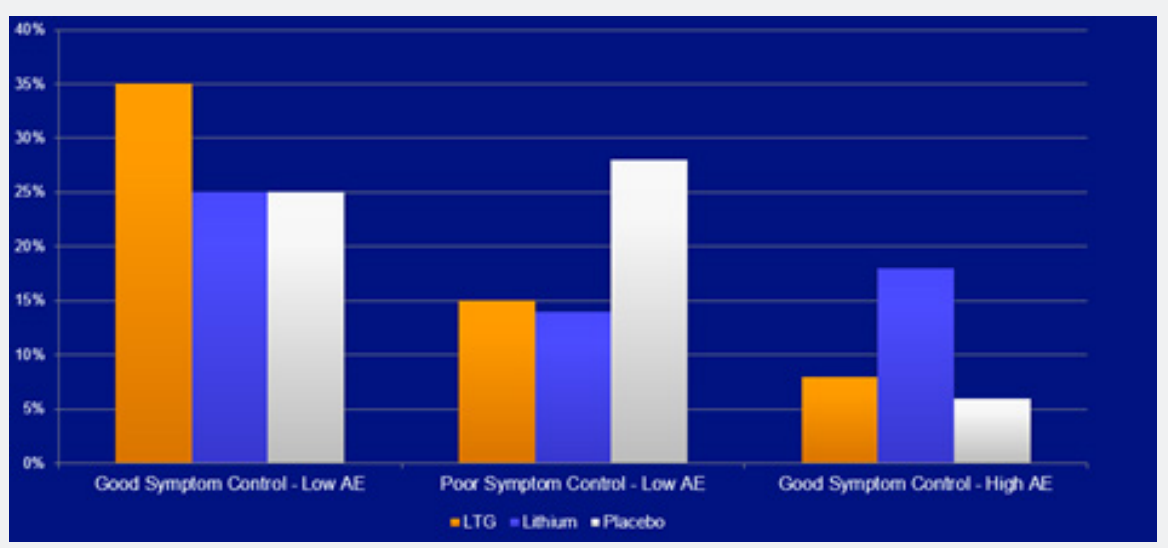

Figure 8: Integrating MOAT symptom states and tolerability by latent class analysis [19].

\section{Summary of Drugs and Studies}

Keeping a patient in treatment is a fundamental goal for long term benefit. Many drugs have evidence of antimanic effects. Few drugs have evidence of depression and maintenance benefits. Tolerability differs substantially among drugs.

\section{References}

1. Angst J, Azorin JM, Bowden CL (2011) Prevalence and characteristics of undiagnosed bipolar disorders in patients with a major depressive episode: the BRIDGE study. Arch Gen Psychiatry 68(8): 791-799.

2. Bowden CL (2008) Bipolar pathophysiology and development of improved treatments. Brain Res. 1235: 92-97.

3. Mallinckrodt CH, Meyers AL, Prakash A, Faries DE, Detke MJ (2007) Simple options for improving signal detection in antidepressant clinical trials. Psychopharmacol Bull 40(2): 101-114.

4. Frye MA (2011) Clinical practice. Bipolar disorder-a focus on depression. N Engl J Med. 364(1): 51-159.

5. Belmaker RH (2007) Treatment of bipolar depression. N Engl J Med 356: 1771-1773.
6. Ghaemi SN1, Ostacher MM, El Mallakh RS, Borrelli D, Baldassano CF, et al. (2010) Antidepressant discontinuation in bipolar depression: a Systematic Treatment Enhancement Program for Bipolar Disorder (STEP-BD) randomized clinical trial of long-term effectiveness and safety. J Clin Psychiatry 71(4): 372-380.

7. Goldberg J (2007) Adjunctive antidepressant use and symptomatic recovery among bipolar depressed patients with concomitant manic symptoms: findings from the STEP-BD. Am J Psychiatry 164(9): 13481355.

8. Gyulai L, Bowden CL, McElroy SL, Calabrese JR, Petty F, et al. (2003) Maintenance efficacy of divalproex in the prevention of bipolar depression. Neuropsychopharmacology 28(7): 1374-1382.

9. Bowden CL, Singh V, Thompson P, Gonzalez JM, Katz MM, et al. (2007) Development of the bipolar inventory of symptoms scale. Acta Psychiatr Scand 116(3): 189-194.

10. V Singh, CL Bowden, JM Gonzalez, P Thompson, TJ Prihoda et al. (2013) Discriminating primary clinical states in bipolar disorder with a comprehensive symptom scale. Acta Psychiatr Scand 127(2): 145-152.

11. CL Bowden, V Singh, R Weisler, P Thompson, X Chang, et al. (2012) Lamotrigine vs. lamotrigine plus divalproex in randomized, placebo- 
controlled maintenance treatment for bipolar depression. Acta Psychiatr Scand 126(5):342-350.

12. Mitchell, Hadzi-Pavlovic, Bowden, et al. (2013) CNS Spectr 18(4): 214 224

13. Judd LL, Akiskal HS, Schettler PJ, Endicott J, Maser J, et al. (2002) Long-term Natural History of Weekly Symptomatic Status of Bipolar 1 Disorder Arch Gen Psychiatry 59(6): 530-537.

14. Turner EH, Matthews AM, Linardatos E, Tell RA, Rosenthal R (2008) Selective Publication of Antidepressant Trials and Its Influence on Apparent Efficacy. N Engl J Med 358: 252-250.

15. Suppes T, Vieta E, Liu S, Brecher M, Paulsson B (2009) Maintenance treatment for patients with bipolar I disorder: results from a north american study of quetiapine in combination with lithium or divalproex (trial 127). Am J Psychiatry 166(4): 476-488.
16. Perugi G, Akiskal HS, Toni C, Simonini E, Gemignani A (2001) The temporal relationship between anxiety disorders and (hypo)mania: a retrospective examination of 63 panic, social phobic and obsessivecompulsive patients with comorbid bipolar disorder. J Affect Disord 67(1-3): 199-206

17. Thompson PM1, Gonzalez JM, Singh V, Schoolfield JD, Katz MM, et al. (2010) Principal domains of behavioral psychopathology identified by the Bipolar Inventory of Signs and Symptoms Scale (BISS). Psychiatry Res 175(3):221-226.

18. Bowden, Gonzalez, Singh, Mintz, Thompson, Bernardo, ECNP, 2009.

19. Bowden C, Tohen M, Mintz J (2015) Multi-state outcome analysis of treatments (MOAT): application of a new approach to evaluate outcomes in longitudinal studies of bipolar disorder. Molecular Psychiatry 21: 237-242.

\section{Your next submission with Juniper Publishers will reach you the below assets}

- Quality Editorial service

- Swift Peer Review

- Reprints availability

- E-prints Service

- Manuscript Podcast for convenient understanding

- Global attainment for your research

- Manuscript accessibility in different formats ( Pdf, E-pub, Full Text, Audio)

- Unceasing customer service

Track the below URL for one-step submission https://juniperpublishers.com/online-submission.php 